Research paper

\title{
Longitudinal characteristics of lymphocyte responses and cytokine profiles in the peripheral blood of SARS-CoV-2 infected patients
}

\author{
Jing Liu ${ }^{\mathrm{a}, \mathrm{c}, 1}$, Sumeng $\mathrm{Li}^{\mathrm{a}, \mathrm{c}, 1}$, Jia Liu ${ }^{\mathrm{a}, \mathrm{c}, 1}$, Boyun Liang ${ }^{\mathrm{a}, \mathrm{c}}$, Xiaobei Wang ${ }^{\mathrm{d}}$, Hua Wang ${ }^{\mathrm{a}}$, \\ Wei $\mathrm{Li}^{\mathrm{a}, \mathrm{c}}$, Qiaoxia Tong ${ }^{\mathrm{a}, \mathrm{c}}$, Jianhua $\mathrm{Yi}^{\mathrm{a}, \mathrm{c}}$, Lei Zhao ${ }^{\mathrm{a}, \mathrm{c}}$, Lijuan Xiong ${ }^{\mathrm{a}, \mathrm{c}}$, Chunxia Guo ${ }^{\mathrm{a}, \mathrm{c}}$, Jin Tian ${ }^{\mathrm{a}, \mathrm{c}}$, \\ Jinzhuo Luo ${ }^{\mathrm{a}, \mathrm{c}}$, Jinghong Yao ${ }^{\mathrm{a}, \mathrm{c}}$, Ran Pang ${ }^{\mathrm{a}, \mathrm{c}}$, Hui Shen ${ }^{\mathrm{a}, \mathrm{c}}$, Cheng Peng, ${ }^{\mathrm{a}, \mathrm{c}}$, Ting Liu ${ }^{\mathrm{a}, \mathrm{c}}$, \\ Qian Zhang ${ }^{\mathrm{a}, \mathrm{c}}$, Jun $\mathrm{Wu}^{\mathrm{a}, \mathrm{c}}$, Ling $\mathrm{Xu}^{\mathrm{a}, \mathrm{c}}$, Sihong $\mathrm{Lu}^{\mathrm{a}, \mathrm{c}}$, Baoju Wang ${ }^{\mathrm{a}, \mathrm{c}}$, Zhihong Weng ${ }^{\mathrm{a}, \mathrm{c}}$ \\ Chunrong Han ${ }^{\mathrm{a}, \mathrm{c}}$, Huabing Zhu ${ }^{\mathrm{a}, \mathrm{c}}$, Ruxia Zhou ${ }^{\mathrm{a}, \mathrm{c}}$, Helong Zhou ${ }^{\mathrm{a}, \mathrm{c}}$, Xiliu Chen ${ }^{\mathrm{a}, \mathrm{c}}$, Pian Ye ${ }^{\mathrm{a}, \mathrm{c}}$, \\ Bin Zhu ${ }^{\mathrm{a}, \mathrm{c}}$, Lu Wang ${ }^{\mathrm{a}, \mathrm{c}}$, Wenqing Zhou ${ }^{\mathrm{a}, \mathrm{Z}}$, Shengsong $\mathrm{He}^{\mathrm{a}, \mathrm{c}}$, Yongwen $\mathrm{He}^{\mathrm{a}, \mathrm{c}}$, Shenghua Jie ${ }^{\mathrm{a}, \mathrm{c}}$,

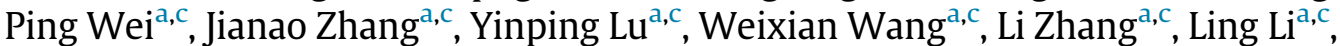 \\ Fengqin Zhou ${ }^{\mathrm{a}, \mathrm{c}}$, Jun Wang ${ }^{\mathrm{b}, \mathrm{c}}$, Ulf Dittmer ${ }^{\mathrm{b}, \mathrm{c}}$, Mengji $\mathrm{Lu}^{\mathrm{b}, \mathrm{c}}$, Yu Hu${ }^{\mathrm{e}, *}$, Dongliang Yang ${ }^{\mathrm{a}, \mathrm{c}, * *}$, \\ Xin Zheng ${ }^{\mathrm{a}, \mathrm{c}, * *}$ \\ a Department of Infectious Diseases, Union Hospital, Tongji Medical College, Huazhong University of Science and Technology, Wuhan 430022, China \\ ${ }^{\mathrm{b}}$ Institute for Virology, University Hospital of Essen, University of Duisburg-Essen, Essen 45147, Germany \\ 'Joint International Laboratory of Infection and Immunity, Huazhong University of Science and Technology, Wuhan 430022, China \\ ${ }^{d}$ Department of Clinical Laboratory, Union Hospital, Tongji Medical College, Huazhong University of Science and Technology, Wuhan 430022, China \\ e Department of Hematology, Union Hospital, Tongji Medical College, Huazhong University of Science and Technology, Wuhan 430022, China
}

\section{A R T I C L E I N F O}

\section{Article History:}

Received 24 February 2020

Revised 5 April 2020

Accepted 6 April 2020

Available online 18 April 2020

Keywords:

Coronavirus

SARS-CoV-2

COVID-19

Lymphopenia

Inflammatory cytokine

\begin{abstract}
A B S T R A C T
Background: The dynamic changes of lymphocyte subsets and cytokines profiles of patients with novel coronavirus disease (COVID-19) and their correlation with the disease severity remain unclear.

Methods: Peripheral blood samples were longitudinally collected from 40 confirmed COVID-19 patients and examined for lymphocyte subsets by flow cytometry and cytokine profiles by specific immunoassays.

Findings: Of the 40 COVID-19 patients enrolled, 13 severe cases showed significant and sustained decreases in lymphocyte counts [0.6 (0.6-0.8)] but increases in neutrophil counts [4.7 (3.6-5.8)] than 27 mild cases [1.1 $(0.8-1.4) ; 2.0(1.5-2.9)]$. Further analysis demonstrated significant decreases in the counts of $\mathrm{T}$ cells, especially CD8 ${ }^{+} \mathrm{T}$ cells, as well as increases in IL-6, IL-10, IL-2 and IFN- $\gamma$ levels in the peripheral blood in the severe cases compared to those in the mild cases. T cell counts and cytokine levels in severe COVID-19 patients who survived the disease gradually recovered at later time points to levels that were comparable to those of the mild cases. Moreover, the neutrophil-to-lymphocyte ratio (NLR) (AUC=0.93) and neutrophil-to$\mathrm{CD}^{+} \mathrm{T}$ cell ratio (N8R) (AUC $=0.94$ ) were identified as powerful prognostic factors affecting the prognosis for severe COVID-19.

Interpretation: The degree of lymphopenia and a proinflammatory cytokine storm is higher in severe COVID19 patients than in mild cases, and is associated with the disease severity. N8R and NLR may serve as a useful prognostic factor for early identification of severe COVID-19 cases.

Funding: The National Natural Science Foundation of China, the National Science and Technology Major Project, the Health Commission of Hubei Province, Huazhong University of Science and Technology, and the Medical Faculty of the University of Duisburg-Essen and Stiftung Universitaetsmedizin, Hospital Essen, Germany. (c) 2020 The Author(s). Published by Elsevier B.V. This is an open access article under the CC BY-NC-ND
\end{abstract} license. (http://creativecommons.org/licenses/by-nc-nd/4.0/)

\footnotetext{
* Corresponding author

** Corresponding authors at: Department of Infectious Diseases, Union Hospital, Tongji Medical College, Huazhong University of Science and Technology, Wuhan 430022, China.

E-mail addresses: huyu@126.com (Y.Hu), dlyang@hust.edu.cn (D. Yang), xin11@hotmail.com (X.Zheng).

${ }^{1}$ These authors contribute equally to this work.
}

\section{Introduction}

First reported in Wuhan, China, on 31 December 2019, an ongoing outbreak of a viral pneumonia in humans has raised acute and grave global concern, and rapidly spread to 197 countries, areas or territories [1]. The causative pathogen was rapidly identified as a novel 


\section{Research in context}

Evidence before this study

Lymphopenia and inflammatory cytokine storm have been shown in severe acute respiratory syndrome coronavirus (SARS-CoV), the Middle East respiratory syndrome coronavirus (MERS-CoV) infections, and coronavirus disease (COVID-19).

\section{Added value of this study}

For the first time, the kinetic changes of lymphopenia, lymphocyte subset and cytokine profile were longitudinally characterized. The significant decreases in the count of $\mathrm{CD}^{+} \mathrm{T}$ cell number and increase in inflammatory cytokine levels (e.g., IL-6, IL-10) are dynamically correlated with the severity of COVID19 patients. T cell counts and cytokine levels in severe COVID19 patients who survived the disease gradually recovered at later time points to levels that were comparable to those of the mild cases. Neutrophil-to-lymphocyte ratio (NLR) (AUC=0.93) and neutrophil-to- $\mathrm{CD}^{+} \mathrm{T}$ cell ratio (N8R) (AUC $=0.94$ ) were identified as powerful prognostic factors affecting the prognosis for severe COVID-19.

\section{Implications of all the available evidence}

The development of severe COVID-19 is the result of imbalanced inflammation and antiviral immune responses. N8R and NLR can serve as useful prognostic factor for early identification of severe COVID-19 cases. This will help physicians to provide timely intervention for COVID-19.

$\beta$-coronavirus, which has since been formally named as the severe acute respiratory syndrome coronavirus 2 (SARS-CoV-2) by the International Committee on Taxonomy of Viruses. According to the World Health Organization coronavirus disease 2019 (COVID-19) situation report and the daily report of the National Health Commission of China, the epidemic of SARS-CoV-2 has so far caused 462,684 confirmed cases (81,968 cases in China, including 11,977 severe cases), and 20,834 deaths (3293 deaths in China) in the world by March 26th, 2020 [1,2]. The disease caused by SARS-CoV-2 has been recently named as the COVID-19 by the World Health Organization. Previous studies about the epidemiological and clinical characteristics of COVID-19 have shown that patients with COVID-19 may develop either mild or severe symptoms of acute respiratory infection, with the mild case patients showing symptoms of fever, dry cough, fatigue, abnormal chest CT findings but with a good prognosis [3,4]. In contrast, some patients develop severe pneumonia, acute respiratory distress syndrome (ARDS) or multiple organ failure, with death rates ranging from between $4.3 \%$ to $15 \%$ according to different study reports [3,5].

Lymphopenia (lymphocyte count $<1.0 \times 10^{9} / \mathrm{L}$ ) 3 and inflammatory cytokine storm are typical laboratory abnormalities observed during highly pathogenic coronavirus infections, such as the severe acute respiratory syndrome coronavirus (SARS-CoV) and the Middle East respiratory syndrome coronavirus (MERS-CoV) infections, and are believed to be associated with disease severities [6,7]. Recent studies have also reported decreases in the counts of lymphocytes (e. g., $\mathrm{CD}^{+} \mathrm{T}$ cell, $\mathrm{CD} 8^{+} \mathrm{T}$ cell) in the peripheral blood and increases in serum inflammatory cytokine levels (e.g., IL-6) in COVID-19 patients $[5,8-10]$. However, it has remained largely unclear in the kinetics of lymphocyte subsets and inflammatory cytokines change in the peripheral blood during COVID-19. In this study, we longitudinally characterized the changes of lymphocyte subsets and cytokines profiles in the peripheral blood of COVID-19 patients with distinct disease severities.

\section{Methods}

\subsection{Data collection}

A written informed consent was regularly obtained from all patients upon admission into Wuhan Union Hospital, China. The study was approved by the Ethics Committee of Wuhan Union Hospital, Tongji Medical College, Huazhong University of Science and Technology, in China. The 40 confirmed COVID-19 patients at Wuhan Union Hospital during January 5 to January 24, 2020 were enrolled into this retrospective single-center study. All medical record information including epidemiological, demographic, clinical manifestation, laboratory data, and outcome data were obtained. All data were checked by a team of trained physicians.

\subsection{Laboratory examination}

Laboratory confirmation of the SARS-CoV-2 was performed by local CDC according to Chinese CDC protocol. Throat-swab specimens were collected from all patients and the samples were maintained in a viral-transport medium for laboratory testing. An infection with other respiratory viruses including influenza A virus, influenza B virus, coxsackie virus, respiratory syncytial virus, parainfluenza virus and enterovirus was excluded by real-time RT-PCR. Specimens, including sputum or bronchoalveolar lavage fluid, blood, urine, and feces, were cultured to identify pathogenic bacteria or fungi that may be associated with the SARS-CoV-2 infection. The specific IgG and IgM of chlamydia pneumonia and mycoplasma pneumonia were detected by chemiluminescence immunoassay. The lymphocyte test kit (Beckman Coulter Inc., FL, USA) was used for lymphocyte subset analysis. Plasma cytokines (IL2, IL4, IL6, IL10, TNF - $\alpha$ and IFN - $\gamma$ ) were detected using the human Th1/2 cytokine kit II (BD Ltd., Franklin lakes, NJ, USA). All tests were performed according to the product manual.

\subsection{Statistical analyses}

Classification variables are expressed in frequency or percentage, and significance was detected by chi square or Fisher's exact test. The quantized variables of parameters are expressed as mean \pm standard deviation, and the significance is tested by $t$-test. Nonparametric variables are expressed in median and quartile intervals, and significance was tested by Mann Whitney U or Kruskal Wallis test. Data (nonnormal distribution) from repeated measures were compared using the generalized linear mixed model. $P<0.05$ was considered statistically significant in all statistical analyses. Principal component analysis (PCA) was performed to identify the major contributing factors among clinical parameters to distinguish between mild and severe cases of COVID-19 patients. The diagnostic values of selected parameters for differentiating between mild and severe cases of COVID-19 patients were assessed by receiver operating characteristic (ROC) and the area under the ROC curve (AUC). SPSS statistical software (Macintosh version 26.0, IBM, Armonk, NY, USA) and R package were used for statistical analysis.

\section{Results}

\subsection{Demographic and clinical characteristics of COVID-19 patients}

The diagnosis of COVID-19 for patients was performed according to the Guidelines of the Diagnosis and Treatment of New Coronavirus Pneumonia (version 5) published by the National Health Commission of China. Mild patients met all following conditions: (1) Epidemiological history, (2) Fever or other respiratory symptoms, (3) Typical CT image abnormities of viral pneumonia, and (4) Positive result of RTPCR for SARS-CoV-2 RNA. Severe patients additionally met at least 
Table 1

Demographics and baseline characteristics of patients infected with SARS-CoV-2.

\begin{tabular}{|c|c|c|c|}
\hline Baseline variables & All patients $(N=40)$ & Mild patients $(N=27)$ & Severe patients $(N=13)$ \\
\hline \multicolumn{4}{|l|}{ Characteristics } \\
\hline Age (year) & $48 \cdot 7 \pm 13.9$ & $43 \cdot 2 \pm 12 \cdot 3$ & $59 \cdot 7 \pm 10 \cdot 1$ \\
\hline \multicolumn{4}{|l|}{ Gender (\%) } \\
\hline Men & $15(37.5)$ & $8(29 \cdot 6)$ & $7(53 \cdot 8)$ \\
\hline Women & $25(62 \cdot 5)$ & $19(70.4)$ & $6(46 \cdot 2)$ \\
\hline Huanan seafood market exposure (\%) & $3(7.5)$ & $1(3 \cdot 7)$ & $2(5.4)$ \\
\hline Current smoking & $5(12 \cdot 5)$ & $3(11 \cdot 1)$ & $2(15.4)$ \\
\hline Underlying diseases (\%) & $14(35 \cdot 0)$ & $7(25.9)$ & $7(53.8)$ \\
\hline Diabetes & $6(15 \cdot 0)$ & $2(7.4)$ & $4(30 \cdot 8)$ \\
\hline Hypertension & $6(15 \cdot 0)$ & $1(3.7)$ & $5(38.5)$ \\
\hline Pituitary adenoma & $2(5 \cdot 0)$ & $1(3.7)$ & $1(7 \cdot 7)$ \\
\hline Thyroid disease & $2(5 \cdot 0)$ & $2(7.4)$ & 0 \\
\hline Malignancy & $2(5 \cdot 0)$ & $2(7.4)$ & 0 \\
\hline Co-infection (\%) & $5(12.5)$ & 0 & $5(38.5)$ \\
\hline Fungi & $4(10 \cdot 0)$ & 0 & $4(30 \cdot 8)$ \\
\hline Bacteria & $1(2 \cdot 5)$ & 0 & $1(7 \cdot 7)$ \\
\hline \multicolumn{4}{|l|}{ Signs and symptoms } \\
\hline Fever & $36(90 \cdot 0)$ & $23(85 \cdot 2)$ & $13(100)$ \\
\hline \multicolumn{4}{|l|}{ Highest temperature, ${ }^{\circ} \mathrm{C}$} \\
\hline$<37.3$ & $4(10 \cdot 0)$ & $4(14 \cdot 8)$ & 0 \\
\hline $37.3-38.0$ & $10(25 \cdot 0)$ & $8(29.6)$ & $2(15.4)$ \\
\hline $38.1-39.0$ & $17(42 \cdot 5)$ & $9(33.3)$ & $8(61.5)$ \\
\hline$>39.0$ & $9(22 \cdot 5)$ & $6(22 \cdot 2)$ & $3(23.1)$ \\
\hline Chill & $10(25 \cdot 0)$ & $5(18.5)$ & $5(38.5)$ \\
\hline Shivering & $5(12 \cdot 5)$ & $2(7.4)$ & $3(23 \cdot 1)$ \\
\hline Fatigue & $22(55 \cdot 0)$ & $14(51.9)$ & $8(61.5)$ \\
\hline Cough & $33(82.5)$ & $22(81.5)$ & $11(84.6)$ \\
\hline Sputum production & $21(52.5)$ & $11(40 \cdot 7)$ & $10(76.9)$ \\
\hline Pharyngalgia & $5(12 \cdot 5)$ & $4(14 \cdot 8)$ & $1(7 \cdot 7)$ \\
\hline Dizziness & $7(17.5)$ & $4(14.8)$ & $3(23 \cdot 1)$ \\
\hline Headache & $8(20.0)$ & $6(22.2)$ & $2(15.4)$ \\
\hline Rhinorrhea & $1(2 \cdot 5)$ & $1(3 \cdot 7)$ & 0 \\
\hline Chest tightness & $12(30 \cdot 0)$ & $7(25.9)$ & $5(38 \cdot 5)$ \\
\hline Chest pain & $1(2 \cdot 5)$ & $1(3.7)$ & 0 \\
\hline shortness of breath & $5(12 \cdot 5)$ & $5(18.5)$ & 0 \\
\hline Dyspnea & $1(2 \cdot 5)$ & $1(3 \cdot 7)$ & 0 \\
\hline Myalgia & $15(37 \cdot 5)$ & $7(25.9)$ & $8(61.5)$ \\
\hline abdominal pain & $1(2 \cdot 5)$ & $1(3 \cdot 7)$ & 0 \\
\hline Diarrhea & $3(7.5)$ & $1(3.7)$ & $2(15.4)$ \\
\hline Nausea & $3(7.5)$ & 0 & $3(23.1)$ \\
\hline Vomiting & $1(2 \cdot 5)$ & 0 & $1(7 \cdot 7)$ \\
\hline Hypoleucocytosis & $10(25 \cdot 0)$ & $8(29.6)$ & $2(15.4)$ \\
\hline Lymphopenia & $23(57.5)$ & $12(44.4)$ & $11(84.6)$ \\
\hline Thrombocytopenia & $5(12.5)$ & $3(11 \cdot 1)$ & $2(15.4)$ \\
\hline Duration of hospitalization (day) & $12 \cdot 6 \pm 6 \cdot 7$ & $12 \cdot 9 \pm 7 \cdot 1$ & $11 \cdot 8 \pm 5 \cdot 9$ \\
\hline $\begin{array}{l}\text { ARDS } \\
\text { prognosis }\end{array}$ & $3(7 \cdot 5)$ & 0 & $3(23 \cdot 1)$ \\
\hline Hospitalization & $4(10)$ & $1(3 \cdot 7)$ & $3(23 \cdot 1)$ \\
\hline Discharge & $33(82.5)$ & $26(96 \cdot 3)$ & $7(53.8)$ \\
\hline Death & $3(7 \cdot 5)$ & 0 & $3(23.1)$ \\
\hline
\end{tabular}

one of the following conditions: (1) Shortness of breath, respiratory rate $\geq 30$ times/min, (2) Oxygen saturation (Resting state) $\leq 93 \%$, or (3) $\mathrm{PaO} 2 / \mathrm{FiO} 2 \leq 300 \mathrm{mmHg}$. A total of 40 patients were enrolled in this study, which were all Wuhan residents and laboratory confirmed cases. The flowchart of patient enrollment is shown in Fig. S5. The patients were divided into two groups according to above-mentioned conditions, including 27 mild cases (67.5\%) and 13 severe cases (32.5\%). Three patients in the severe group died on day 15,18 and 21 after disease onset.

The enrolled COVID-19 patients consisted of 15 males (37.5\%) and 25 females (62.5\%) (Table 1$)$. Only 3 patients (7.5\%) had an exposure history (shopping) to the Huanan seafood market in Wuhan. The medium age of the patients was $48.7 \pm 13.9$ years old. The ages of the severe patient group $(59.7 \pm 10 \cdot 1$ years) were older than that of the mild group ( $43.2 \pm 12.3$ years). The duration of hospitalization was $12.6 \pm 6.7$ day, and three [7.5\%] of the patients died. A total of 14 (35\%) patients in both groups had underlying chronic medical conditions, including diabetes (6 [15\%]), hypertension (6 [15\%]), pituitary adenoma (2 [5\%]), thyroid disease $(2[5 \%])$ and tumor disease $(2[5 \%])$.
Four severe patients had mixed fungal infection and 1 severe patient had a mixed bacterial infection (Table 1). All severe patients and $85.2 \%$ of the mild patients had fever, while no significant difference in the degrees of temperature was observed between the two groups (Table 1 ). The severe patients showed significantly higher frequencies in the occurrence of sputum production, myalgia and nausea. Three patients in the severe group developed (acute respiratory distress syndrome, ARDS). All patients received antiviral treatment, including interferon $\alpha 2 \mathrm{~b}(16,40.0 \%)$, ribavirin (24, 60.0\%), abidol (18, 45.0\%), and/or oseltamivir $(17,42.5 \%)$, as well as antibiotic treatment, including moxifloxacin $(40,100 \%)$, cephalosporins $(20,50 \%)$, penicillin (1, $2.5 \%$ ), and/or other antibiotics (29, $72.5 \%$ ). Seventeen (42.5\%) patients received antifungal treatment (13 cases took prophylactic treatment) and $8(20 \%)$ received methylprednisolone therapy (1 mild case and 7 severe cases) (Table S1). The levels of fibrinogen, D-dimer, total bilirubin, aspartate transaminase, alanine transaminase, lactate dehydrogenase, creatine kinase, C-reactive protein (CRP), ferritin and serum amyloid A protein (SAA) in the peripheral blood of the severe patients were significantly higher at admission compared to the mild 
Table 2

\begin{tabular}{|c|c|c|c|}
\hline Baseline variables & All patients $(N=40)$ & $\begin{array}{l}\text { Mild patients } \\
(N=27)\end{array}$ & $\begin{array}{l}\text { Severe patients } \\
(N=13)\end{array}$ \\
\hline Hemoglobin (g/l) & $126 \cdot 4 \pm 13.4$ & $127 \cdot 8 \pm 13 \cdot 1$ & $123 \cdot 4 \pm 14.0$ \\
\hline Platelet $\left(\times 10^{9} / \mathrm{L}\right)$ & $183 \cdot 1 \pm 69 \cdot 0$ & $181.4 \pm 70.7$ & $186 \cdot 6 \pm 68 \cdot 1$ \\
\hline $\begin{array}{l}\text { White blood cell }(\times \\
\left.10^{9} / \mathrm{L}\right)\end{array}$ & $4 \cdot 8 \pm 2 \cdot 6$ & $3 \cdot 9 \pm 1 \cdot 5$ & $6 \cdot 6 \pm 3 \cdot 4$ \\
\hline Neutrophil $\left(\times 10^{9} / \mathrm{L}\right)$ & $2 \cdot 8(1.6-4 \cdot 3)$ & $2 \cdot 0(1.5-2 \cdot 9)$ & $4.7(3.6-5.8)$ \\
\hline $\begin{array}{l}\text { Lymphocyte }\left(\times 10^{9} /\right. \\
\text { L) }\end{array}$ & $0.9(0.7-1.3)$ & $1.1(0.8-1.4)$ & $0.6(0.6-0.8)$ \\
\hline Monocyte $\left(\times 10^{9} / \mathrm{L}\right)$ & $0.3(0.2-0.5)$ & $0.3(0.2-0.5)$ & $0.2(0.2-0.5)$ \\
\hline TBil (umol/l) & $10 \cdot 3 \pm 5 \cdot 0$ & $8 \cdot 8 \pm 4 \cdot 1$ & $13 \cdot 2 \pm 5 \cdot 5$ \\
\hline $\operatorname{ALT}(\mathrm{U} / \mathrm{L})$ & $22.5(16 \cdot 8-31 \cdot 2)$ & $19.0(13.5-26.0)$ & $27.0(23.0-50 \cdot 0)$ \\
\hline AST (U/L) & $34 \cdot 1 \pm 17 \cdot 7$ & $25.9 \pm 9.5$ & $51 \cdot 2 \pm 18 \cdot 7$ \\
\hline $\mathrm{LDH}(\mathrm{U} / \mathrm{L})$ & $303 \cdot 9 \pm 168 \cdot 8$ & $221 \cdot 5 \pm 71 \cdot 2$ & $462 \cdot 4 \pm 190 \cdot 6$ \\
\hline $\mathrm{CK}(\mathrm{U} / \mathrm{L})$ & $59 \cdot 5(45 \cdot 0-88 \cdot 8)$ & $51.0(45.0-68.0)$ & $104.0(77 \cdot 0-124.0)$ \\
\hline $\begin{array}{l}\text { Blood urea nitrogen } \\
(\mathrm{mmol} / \mathrm{l})\end{array}$ & $3 \cdot 2(2 \cdot 5-4 \cdot 3)$ & $3 \cdot 2(2 \cdot 5-4 \cdot 4)$ & $3.3(2.7-3.7)$ \\
\hline $\begin{array}{l}\text { Serum creatinine } \\
\quad(\mathrm{umol} / \mathrm{l})\end{array}$ & $67 \cdot 3 \pm 19 \cdot 7$ & $64.0 \pm 13.3$ & $74 \cdot 2 \pm 28 \cdot 3$ \\
\hline $\begin{array}{l}\text { Blood potassium } \\
(\mathrm{mmol} / \mathrm{l})\end{array}$ & $3 \cdot 8 \pm 0 \cdot 5$ & $3 \cdot 9 \pm 0 \cdot 5$ & $3.7 \pm 0.4$ \\
\hline $\begin{array}{l}\text { Blood sodium } \\
(\mathrm{mmol} / \mathrm{l})\end{array}$ & $145 \cdot 9 \pm 43 \cdot 4$ & $149 \cdot 5 \pm 52 \cdot 5$ & $138 \cdot 6 \pm 6 \cdot 2$ \\
\hline D-Dimer (mg/l) & $0.6(0.3-0.9)$ & $0.4(0.2-0.8)$ & $0.9(0.7-1.5)$ \\
\hline $\mathrm{PT}(\mathrm{s})$ & $13 \cdot 2 \pm 0.6$ & $13 \cdot 1 \pm 0.6$ & $13.4 \pm 0.6$ \\
\hline APTT (s) & $39.5 \pm 4.5$ & $39.5 \pm 4.6$ & $39.5 \pm 4.2$ \\
\hline INR & $1 \cdot 0 \pm 0 \cdot 1$ & $1.0 \pm 0.1$ & $1.0 \pm 0.1$ \\
\hline FIB $(g / l)$ & $5 \cdot 1 \pm 1 \cdot 6$ & $4.5 \pm 1.4$ & $6 \cdot 3 \pm 1 \cdot 3$ \\
\hline $\operatorname{IgE}$ & $43.9(14.4-98.0)$ & $26 \cdot 5(12 \cdot 8-76 \cdot 1)$ & $43.9(27.0-105.5)$ \\
\hline $\lg G$ & $11 \cdot 1 \pm 2 \cdot 0$ & $10 \cdot 8 \pm 2 \cdot 0$ & $11.5 \pm 2.0$ \\
\hline $\operatorname{Ig} A$ & $2.2 \pm 0.7$ & $2 \cdot 2 \pm 0.8$ & $2.4 \pm 0.6$ \\
\hline $\operatorname{IgM}$ & $1.1 \pm 0.4$ & $1 \cdot 1 \pm 0.5$ & $1.1 \pm 0.3$ \\
\hline $\begin{array}{l}\text { C-reactive protein } \\
(\mathrm{mg} / \mathrm{l})\end{array}$ & $38.1(4.7-65 \cdot 2)$ & $7 \cdot 6(3 \cdot 1-57 \cdot 3)$ & $62 \cdot 9(42 \cdot 4-86 \cdot 6)$ \\
\hline Ferritin (ug/l) & $\begin{array}{l}596.5 \\
\quad(308.6-1087.6)\end{array}$ & $\begin{array}{l}367.8 \\
\quad(174.7-522 \cdot 0)\end{array}$ & $\begin{array}{l}835.5 \\
\quad(635.4-1538.8)\end{array}$ \\
\hline $\mathrm{SAA}(\mathrm{mg} / \mathrm{l})$ & $134.4(35.7-586 \cdot 3)$ & $46 \cdot 9(20 \cdot 5-134 \cdot 4)$ & $\begin{array}{l}607.1 \\
\quad(381 \cdot 9-686 \cdot 2)\end{array}$ \\
\hline $\mathrm{C} 3(\mathrm{~g} / \mathrm{l})$ & $0.8 \pm 0.2$ & $0.8 \pm 0.2$ & $0.8 \pm 0.1$ \\
\hline $\mathrm{C} 4(\mathrm{~g} / \mathrm{l})$ & $0 \cdot 3 \pm 0 \cdot 1$ & $0.3 \pm 0 \cdot 1$ & $0.3 \pm 0 \cdot 1$ \\
\hline
\end{tabular}

patients (Table 2). No significant differences in the serum levels of immunoglobulins (IgA, IgG and IgM), complement C3 or C4 were observed between the two groups (Table 2). We also analyzed the impact of methylprednisolone treatment on the lymphocyte and neutrophil counts, as well as other cytokine levels. The results showed that patients with methylprednisolone treatment had higher lymphocyte counts, lower neutrophil counts, NLR, N8R and serum IL6 levels than patients without methylprednisolone treatment. However, the differences were not statistically significant (Table S2).

\subsection{Kinetic analysis of lymphocyte subsets in the peripheral blood of COVID-19 patients}

Lymphopenia was observed in $44.4 \%(12 / 27)$ of mild patients and $84.6 \%(11 / 13)$ of severe patients at the onset of the disease. As shown in Table 2, the absolute counts of lymphocytes in the peripheral blood of the severe patients was significantly lower, while the absolute counts of total white blood cells (WBCs) and neutrophils were significantly higher than those of the mild patients at the time of hospital admission. No significant difference in monocyte counts was observed between the two groups (Table 2). Next, we analyzed the kinetic changes of WBCs, neutrophils and monocytes as well as different lymphocyte subsets in the peripheral blood of COVID-19 patients from the disease onset to at least 16 days later. The three mortalities in the severe group were excluded from the analysis due to the lack of kinetic data. Significant increases in total WBCs counts in the severe group were only observed at the time point of onset (within 3 days) but not during the following period of disease progression compared to the mild group (Fig. 1a). Significant increases in the neutrophil counts of the severe group were observed not only at the time point of disease onset, but also at 13-15 days later compared to the mild group (Fig. 1b). In contrast, a sustained decrease in the lymphocyte counts of the severe group was observed compared to those of the mild patients. The difference was significant at the time point of disease onset and became even greater on 4-6 days later (Fig. 1c). From 7 to 15 days after disease onset, the lymphocyte counts gradually increased in the severe group, and reached a comparable level to that of the mild patients at 16 days after disease onset (Fig. 1c). No significant differences in monocyte counts were observed between the two groups during the whole observation period (Fig. 1d). There were 4 patients co-infected with fungi in the severe case group. To examine the impact of fungal infection on the results, we performed the analyses by excluding the data of the 4 cases with fungal co-infections from the severe patient group, and similar kinetic changes of different subsets of PBMCs were observed when these 4 cases were excluded. Total WBCs counts in the severe group significantly increased at the time point of onset (within 3 days) but not during the following period of disease progression compared to the mild group (Fig. S4a). Significant increases in the neutrophil counts of the severe group were observed only at the time point of disease onset (Fig. S4b). In contrast, significant decreases in lymphocyte counts of the severe group were observed at the time point of disease onset and became even greater on 4-6 days later compared to those of the mild patients (Fig. S4c). No significant differences in monocyte counts were observed between the two groups during the whole observation period (Fig. S4d). 


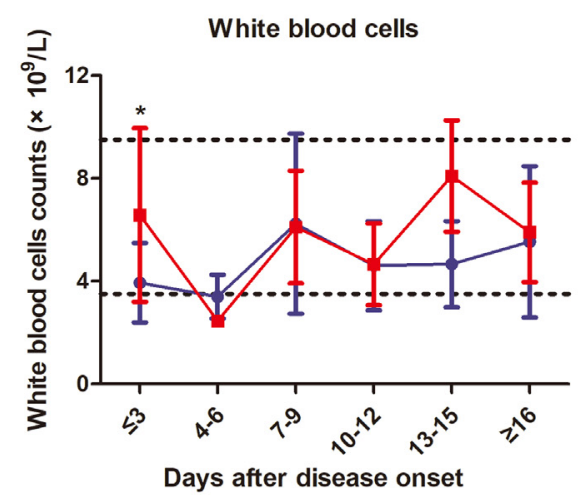

c

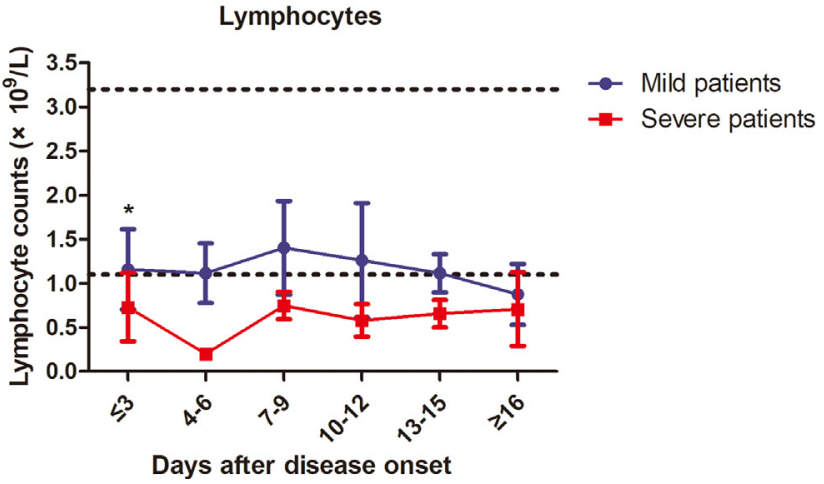

$\mathrm{b}$

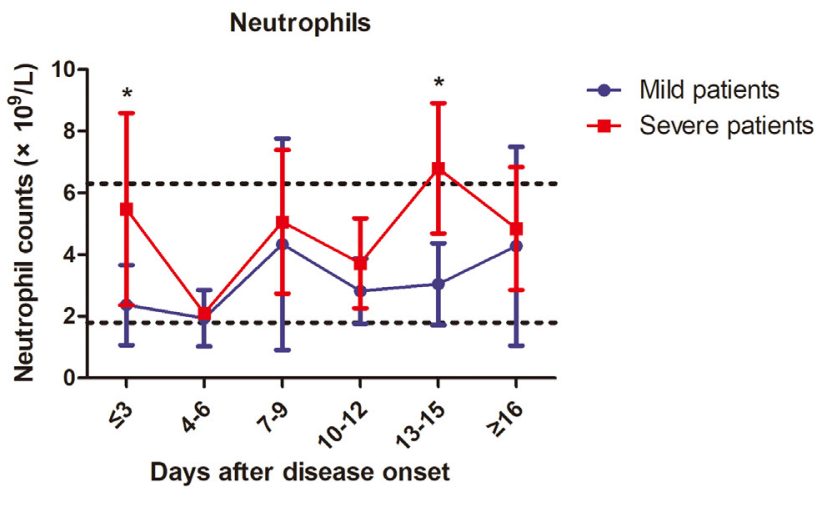

d

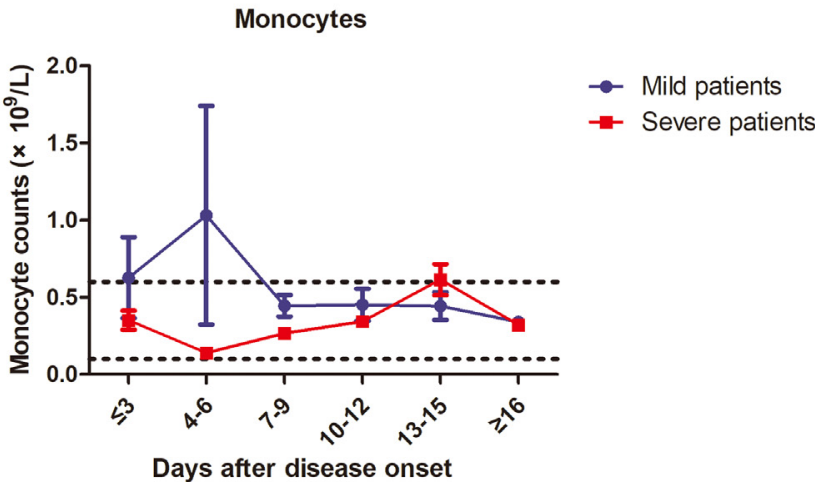

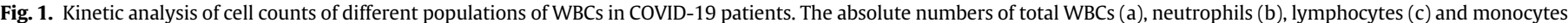

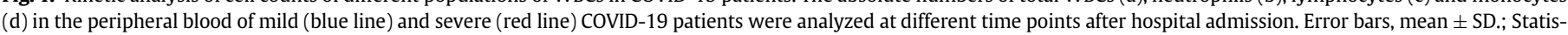

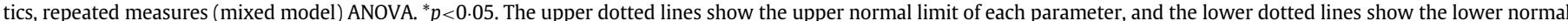

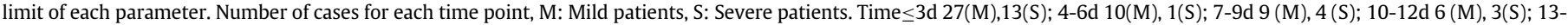
$15 \mathrm{~d} 4(\mathrm{M}), 6(\mathrm{~S}) ; \geq 16 \mathrm{~d} 2(\mathrm{M}), 7(\mathrm{~S})$.

In order to further determine the kinetic changes of different lymphocyte subsets in the peripheral blood of COVID-19 patients, we performed flow cytometry to stain $\mathrm{CD}^{+} \mathrm{T}$ cells, $\mathrm{CD} 4^{+}$and $\mathrm{CD}^{+} \mathrm{T}$ cell subsets, B cells and NK cells. Similar to the findings for lymphocytes, sustained decreases in $\mathrm{CD}^{+}, \mathrm{CD}^{+}$and $\mathrm{CD}^{+}{ }^{+} \mathrm{T}$ cell counts were observed in the severe group compared to those of the mild patients during clinical observation (Figs. $2 \mathrm{a}-\mathrm{c}$ and $\mathrm{S} 1$ ). The lowest $\mathrm{CD} 3^{+}, \mathrm{CD}^{+}$ and $\mathrm{CD}^{+} \mathrm{T}$ cell counts were observed at 4-6 days after disease onset (Fig. 2a-c). The differences in $\mathrm{CD}^{+}$and $\mathrm{CD}^{+} \mathrm{T}$ cell counts between the two groups were significant at the time point of disease onset and 7-9 days later (Fig. 2a and c). However, the differences in $\mathrm{CD} 4^{+} \mathrm{T}$ cell counts between the two groups did not reach a statistical significance at any time point (Fig. 2c). The T cell counts started to gradually increase in the severe group starting at 7 days after disease onset, and reached comparable levels to those in the mild patients on day 16 after disease onset (Fig. 2a-c). No significant differences in B cell and NK cell counts were observed between the two groups during the whole course of the disease (Fig. $2 \mathrm{~d}$ and e).

\subsection{Kinetic analysis of inflammatory cytokine levels in the serum of COVID-19 patients}

A previous study demonstrated changes in inflammatory cytokine levels, such as IL-2, IL-7, IL-10, and TNF- $\alpha$, in the serum of COVID-19 patients [3]. Therefore, we further characterized the kinetic changes of inflammatory cytokine levels, including IL-2, IL-4, IL-6, IL-10, IFN- $\gamma$ and TNF- $\alpha$, in the serum of our patient cohort. Fluctuations in the serum levels of these cytokines in the mild patient group were minor. In contrast, the severe patient group showed more significant fluctuations in the serum levels of these cytokines (Fig. 3). All examined cytokines, except IL-6, reached their peak levels in the serum at
3-6 days after disease onset (Fig. 3). Both IL-6 and IL-10 levels showed sustained increases in the severe group compared to the mild group (Fig. 3a and b). Reductions in serum IL-6 levels in the severe group started at 16 days after disease onset, and IL-10 levels were lowest at 13 days after disease onset (Fig. 3a and b). Significant increases in serum IL- 2 and IFN- $\gamma$ levels in the severe group were only observed at 4-6 days after disease onset (Fig. $3 \mathrm{c}$ and f). No significant differences in IL- 4 and TNF- $\alpha$ levels were observed between the two groups during the whole course of the disease (Fig. $3 \mathrm{~d}$ and e). All examined cytokines reached similar levels between the severe and mild patient groups at 16 days after disease onset (Fig. 3). We observed higher CRP levels in the severe group than the mild group at most time points; however, the differences were not significant (Fig. S6). Moreover, we also performed the analyses by excluding the 4 patients with fungal co-infections from the severe patient group. We observed that both IL- 6 and IL-10 levels showed sustained increases in the severe group compared to the mild group (Fig. S3a and b). Significant increases in serum IFN- $\gamma$ levels in the severe group were only observed at 4-6 days after disease onset (Fig. S3f). No significant differences in IL-2, IL4 and TNF- $\alpha$ levels were observed between the two groups during the whole course of the disease (Fig. $\mathrm{S} 3 \mathrm{c}-\mathrm{e})$.

\subsection{Prognostic factors for the identification of severe COVID-19 cases}

Next, we examined the possibilities of using above-mentioned parameters as prognostic factors for identifying severe cases in COVID-19 patients. PCA was firstly performed by R package "factoextra" to identify correlated variables for distinguishing severe patients from mild patients (Fig. 4a). Four most contributing variables, neutrophil-to-CD8 ${ }^{+} \mathrm{T}$ cell ratio (N8R), neutrophil-to-lymphocyte ratio (NLR), 

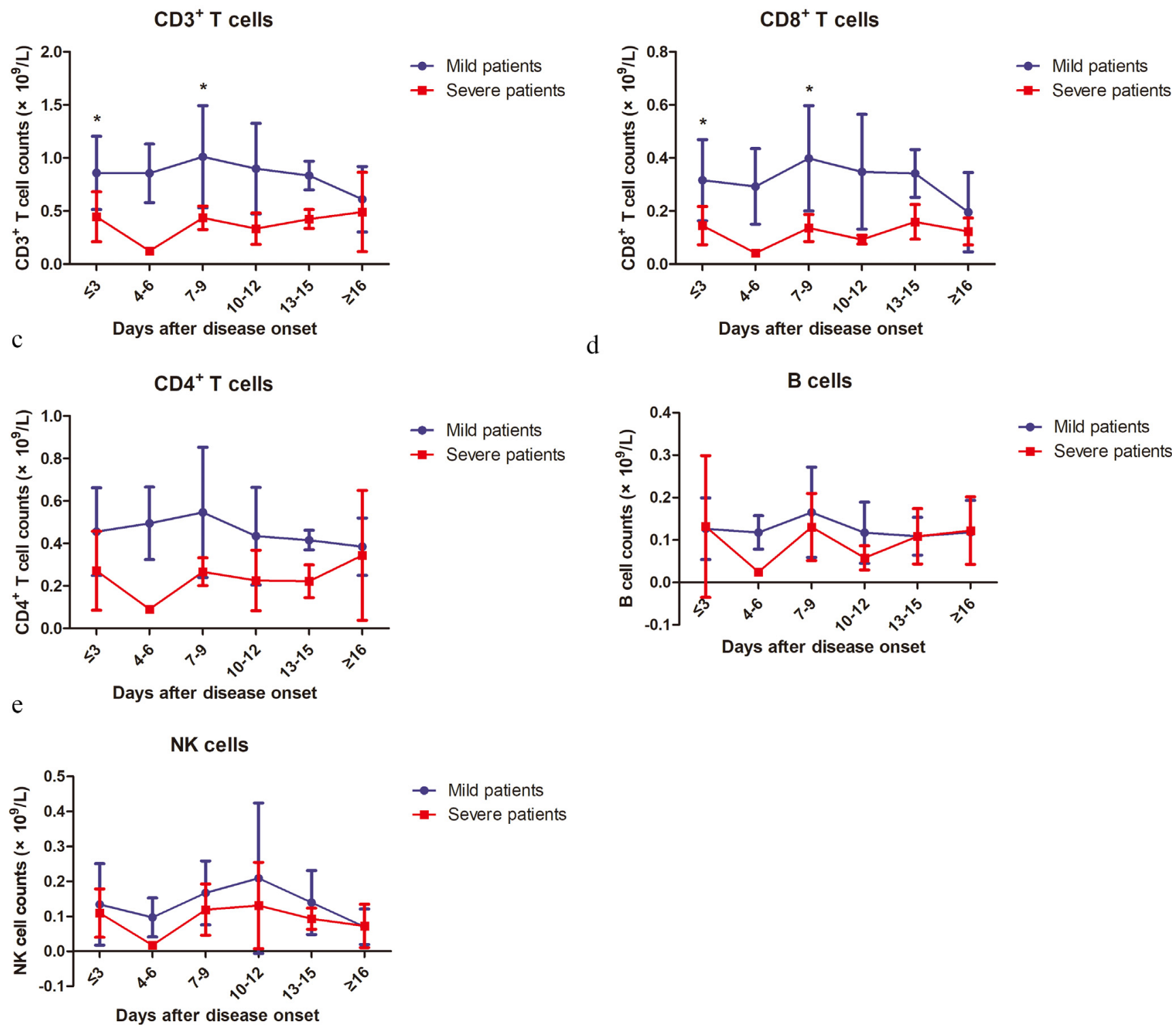

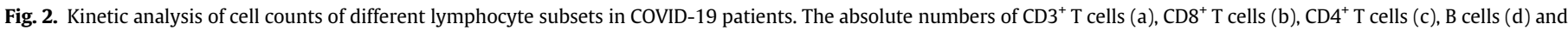

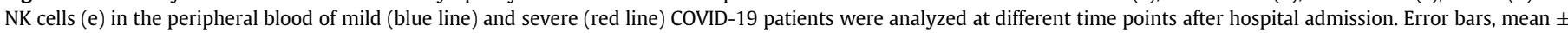

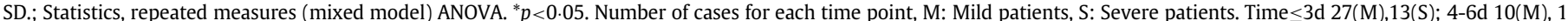
(S); 7-9d 9 (M), 4 (S); 10-12d 6 (M), 3(S); 13-15d 4 (M), 6 (S); $\geq 16 \mathrm{~d} 2$ (M), 7 (S).

neutrophil counts (NEC) and white blood cells counts (WBCC) were selected as potential prognostic factors for further detailed statistical analysis. To assess the diagnostic value of these four selected parameters, the receiver operating characteristic (ROC) curve and the area under ROC curve (AUC) were calculated by R package "pROC" (Fig. 4b). The results of this analysis identified N8R with a higher AUC (0.94) than NLR (0.93), NE (0.91) and WBC (0.85) (Fig. 4b). Simultaneously, the cutoff values were calculated from the ROC curves, with a value of 21.9 for N8R (Specificity: $92.6 \%$, Sensitivity: $84.6 \%$ ), 5.0 for $\operatorname{NLR}(96.3 \%, 84.6 \%), 3.2$ for NE $(81.5 \%, 84.6 \%)$ and 4.3 for WBC $(74.1 \%$, $84.6 \%)$. The further univariate analysis including the four factors of $\mathrm{N} 8 \mathrm{R}>21.9, \mathrm{NLR}>5.0, \mathrm{NE}>3.2$ and $\mathrm{WBC}>4.3$ were used to calculate odds ratios (ORs). The results were obtained for NLR (OR: 143, 95\% Cl: 11.72-1745.3), N8R (OR: 68.75, 95\% Cl: 8.55-552.68), NE (OR: 22, 95\% Cl: 3.646-132.735) and WBC (OR: 55, 95\% Cl: 6.779-446.23) within our patient cohort were selected as predictive factors for severe COVID-19. We also performed a 5-fold cross-validation in all patients by using univariate logistic regression for each variable. The average of the AUC and the standard deviation are listed below: N8R: $0.908 \pm 0.145$, NLR: $0.935 \pm 0.093$, WBCC: $0.868 \pm 0.141$, NEC: $0.828 \pm 0.198$. The predictive performances of N8R and NLR were still significant when fungal co-infection cases were excluded from the group (Fig. S2b).

\section{Discussion}

In this study, we analyzed the clinical features and immunological characteristics of peripheral blood in patients with COVID-19. Although the majority of the patients did not have an exposure history to the Huanan seafood market in Wuhan, the clinical characteristics of these patients are very similar to those reported in previous studies [3,5,11]. In severe patients, the ages as well as the proportion of underlying diseases are higher, and co-infection also occurs. Recent reports show that the lymphocyte counts are normal in COVID-19 patients with mild diseases. In contrast, $63 \%-70.3 \%$ of patients with severe diseases have lymphopenia and the lymphocyte 
IL-6

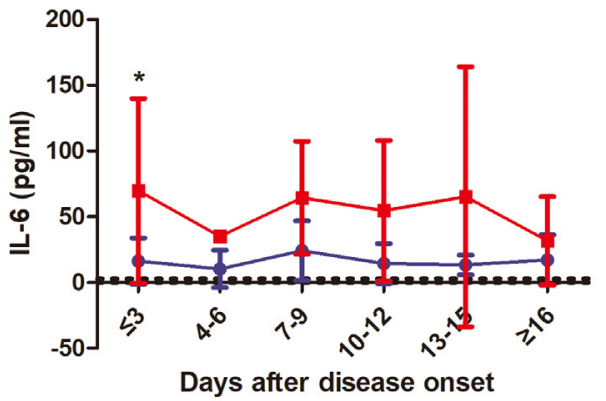

c

IL-2

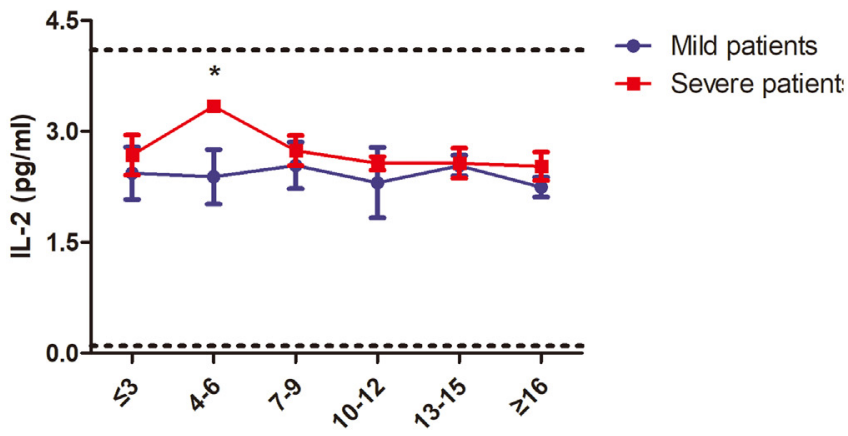

Days after disease onset

e

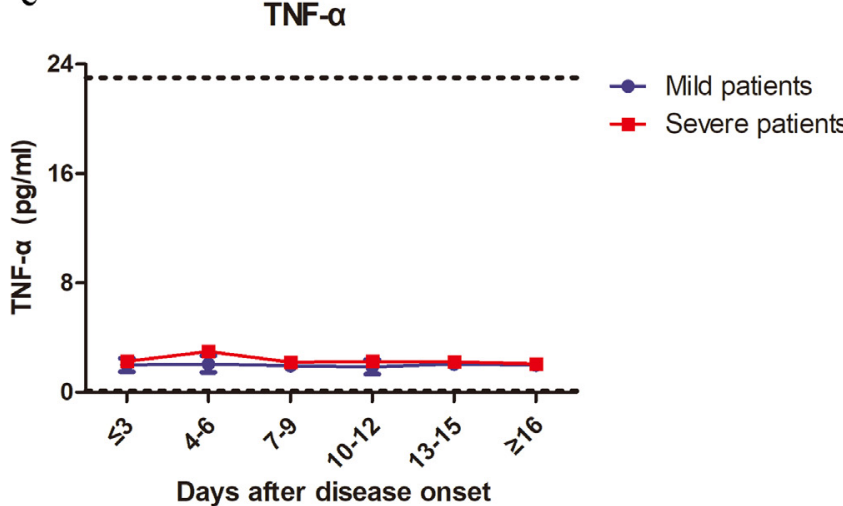

$\mathrm{b}$

IL-10

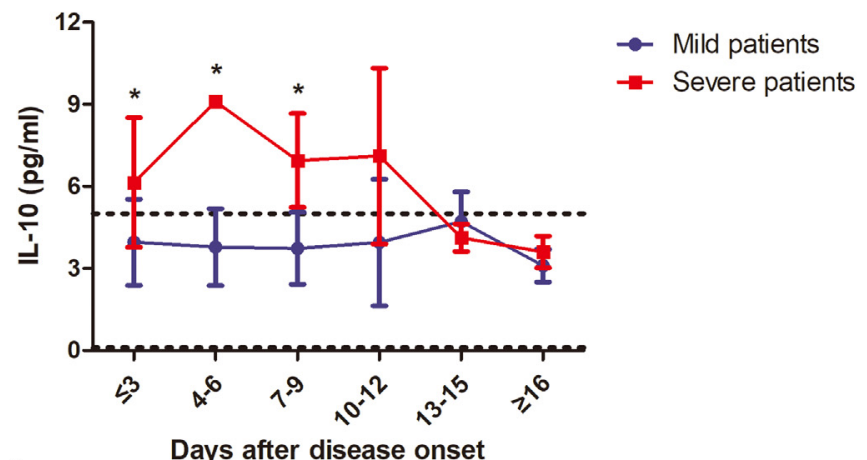

d

IL-4

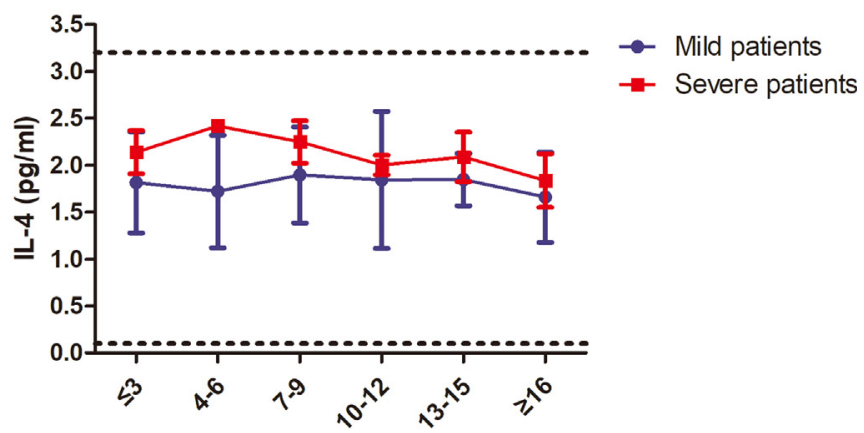

Days after disease onset

f

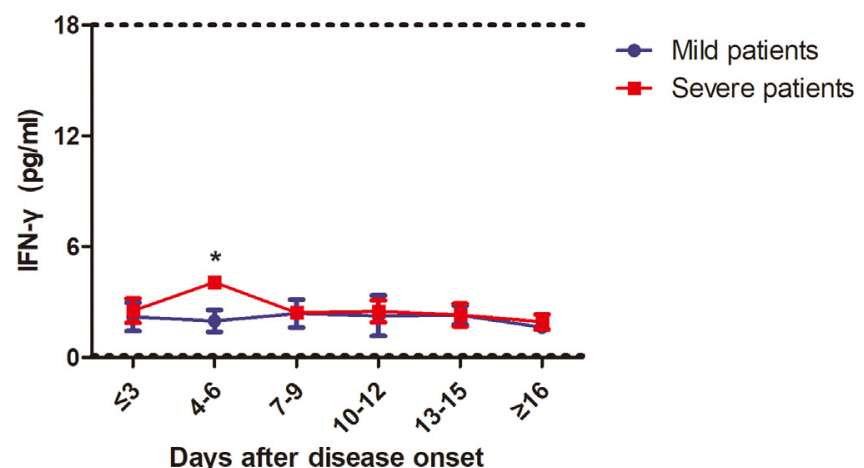

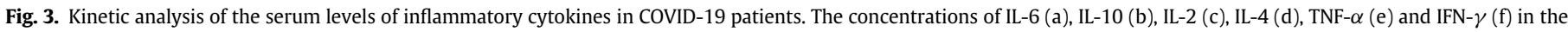

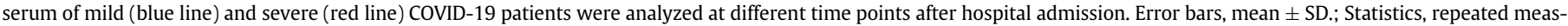

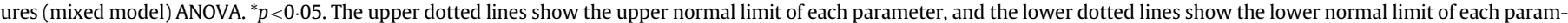

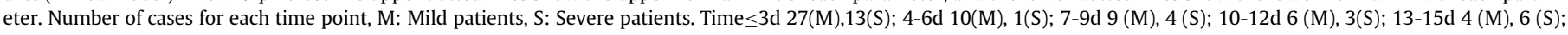
$\geq 16 \mathrm{~d} 2$ (M), 7 (S).

counts in patients with a mortal outcome remain at a low level $[5,11]$. Our study also confirmed higher rates of developing lymphopenia in severe patients than in mild patients ( $84.6 \%$ vs. $44.4 \%$ ). Recent studies reported that the SARS-CoV-2 infection may primarily affect $\mathrm{T}$ lymphocytes particularly $\mathrm{CD}^{+}{ }^{+}$and $\mathrm{CD}^{+} \mathrm{T}$ cells, which might be highly involved in the pathological process of COVID-19 [10]. We also found that the development of lymphopenia in severe patients was mainly related to the significantly decreased absolute counts of $\mathrm{T}$ cells, especially $\mathrm{CD}^{+} \mathrm{T}$ cells, but not to the absolute counts of B cells and NK cells. The decrease of $T$ cells in the severe patient group reached its peak within the first week during the disease course, and then T cell numbers gradually increased during the second week and recovered to a comparable level to that of the mild patient group in the third week. All the severe patients included in our study survived the disease, and thus we speculate this course is associated with a favorable outcome in severe COVID-19 patients.

Previous studies have shown that elevated levels of proinflammatory cytokines, such as IFN- $\gamma$, TNF-a, IL- 6 and IL-8, are associated with severe lung injury and adverse outcomes of SARS-CoV or MERS-CoV infection [7,12-14]. Our results also demonstrate that severe COVID19 patients have higher concentrations of IL6, IL10, IL2 and IFN- $\gamma$ in the serum than mild cases, suggesting that the magnitude of cytokine storm is associated with the disease severity. Additionally, T cells are important for dampening overactive innate immune responses during viral infection $[15,16]$. Thus, a loss of T cells during SARS-CoV-2 infection may result in aggravated inflammatory responses, while a 
a

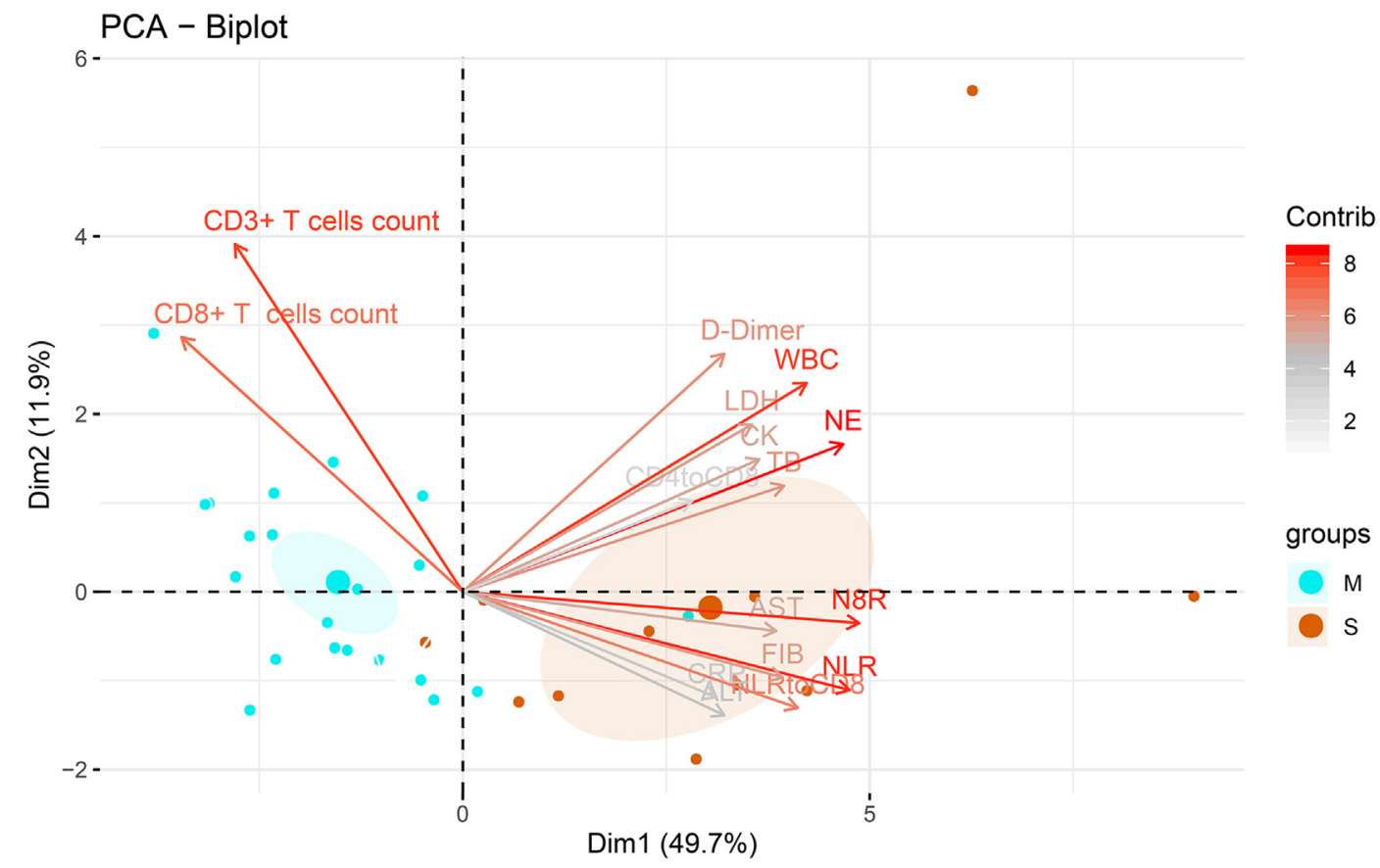

b
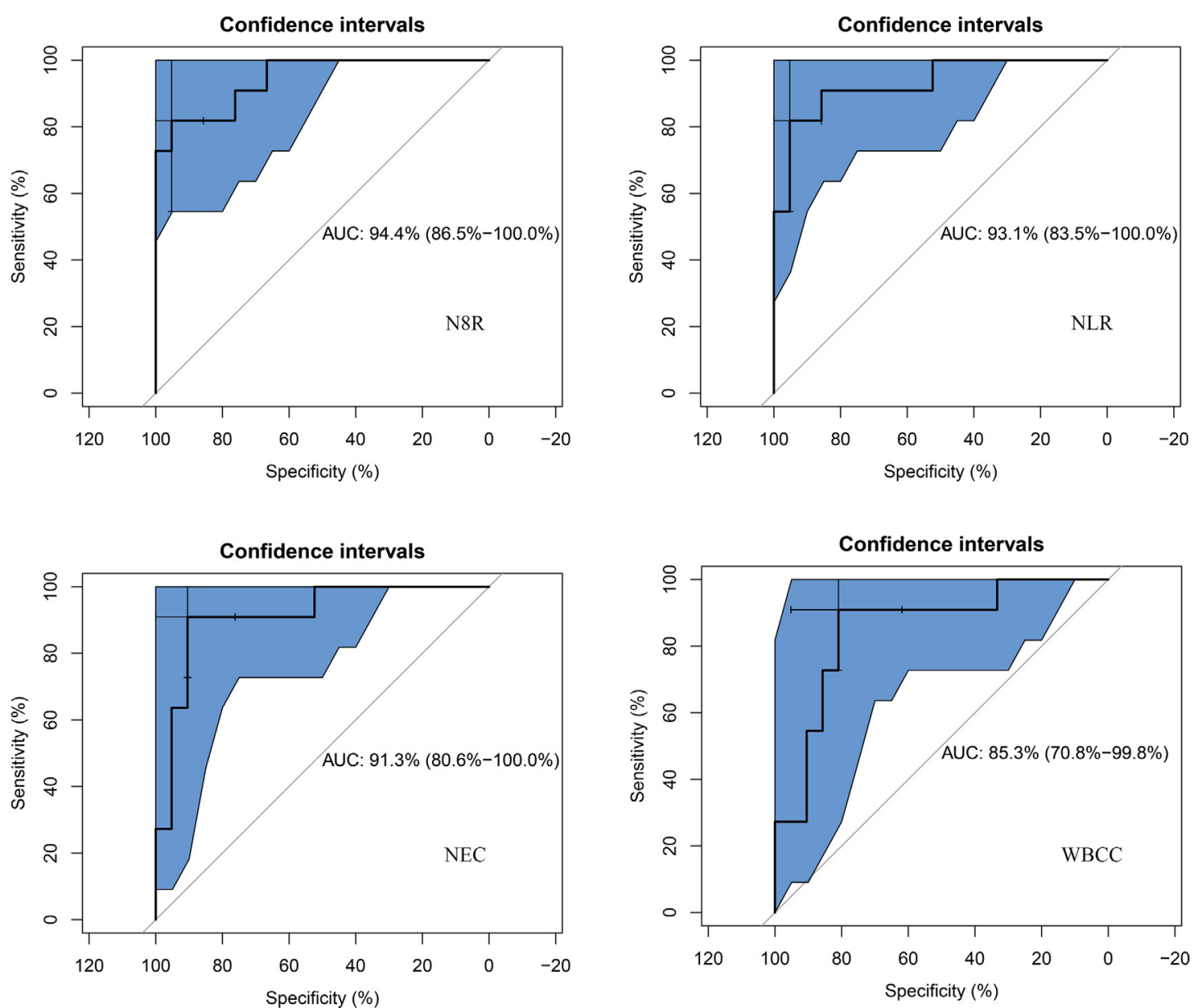

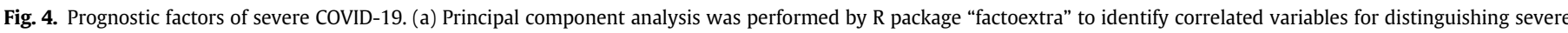

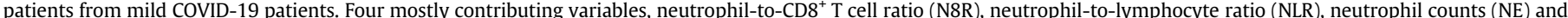

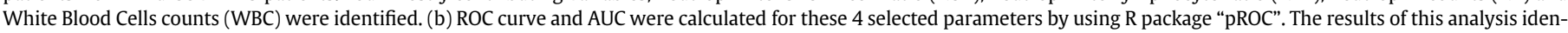
tified N8R with a higher AUC (0.94) than NLR (0.93), NE (0.91) and WBC (0.85). 
restoration of $\mathrm{T}$ cell numbers may alleviate them. In line with this hypothesis, we observed that the kinetic changes of $T$ cell counts are reversely correlated with the kinetic changes of most examined cytokine levels in the peripheral blood in severe COVID-19 patients. While T cell counts drop to their lowest levels at 4-6 days after disease onset, serum IL-10, IL-2, IL- 4 , TNF- $\alpha$ and IFN- $\gamma$ levels reach their peaks. The courses of restoring $\mathrm{T}$ cell numbers are associated with decreases of serum IL-6, IL-10, IL-2, IL-4, TNF- $\alpha$ and IFN- $\gamma$ levels.

The early identification of risk factors for severe COVID-19 patients may facilitate appropriate supportive care and prompt access to the intensive care unit if necessary. A recent study in a 61patient cohort reported that the NLR was the most useful prognostic factor affecting the prognosis for severe COVID-19 [17], and immunological markers (e.g., $\mathrm{CD} 4^{+} \mathrm{T}$ cell, $\mathrm{CD} 8^{+} \mathrm{T}$ cell, NLR) tend to be an independent predictor for COVID-19 severity and treatment efficacy $[9,18]$. The severity of pathological injury during SARS or MERS correlates with the extensive infiltration of neutrophils in the lung and increased neutrophil numbers in the peripheral blood [19]. Thus, the magnitude of increase in neutrophil counts may suggest the intensity of inflammatory responses in COVID-19 patients. Besides, the magnitude of the decrease in lymphocyte counts also indicates the extent of the impairment of immune system by the viral infection. Therefore, NLR may serve as a useful factor to reflect the intensity of imbalance of inflammation and immune responses in COVID-19 patients. In this study, we also screened the potential prognostic factors affecting the incidence of severe illness in our patient cohort. Based on our findings with analyzing lymphocyte subsets, we further included the ratio of neutrophils to different lymphocyte subsets as parameters. Our kinetic analysis revealed that $\mathrm{CD}^{+} \mathrm{T}$ cells are the major lymphocyte subset which decreases in cell numbers during COVID-19. In line with this finding, our results demonstrate that N8R has a comparable performance with NLR in the ROC curve analysis, and both N8R and NLR may serve as powerful factors for predicting the severe illness incidence in COVID patients.

In summary, our study of the immunological characteristics of the peripheral blood in COVID-19 patients shows that the numbers of neutrophils and T cells, especially $\mathrm{CD}^{+} \mathrm{T}$ cells, as well as the levels of inflammatory cytokines in the peripheral blood is dynamically correlated with the severity of the disease. To the best of our knowledge, this is the first work to describe the kinetic changes of lymphocyte subsets and cytokine profiles in COVID-19 patients. Importantly, we identified N8R and NLR as powerful prognostic factors for the early identification of severe COVID-19 cases. This work may help to achieve a better understanding of immune function disorder as well as immunopathogenesis during SARS-CoV-2 infection, and help physicians to provide timely intervention for COVID- 19 .

\section{Declaration of Competing Interest}

The authors disclose no conflicts of interest.

\section{Acknowledgment}

We thank all the doctors, nurses, disease control workers, and researchers who have fought bravely and ceaseless against the virus at the frontline during the SARS-CoV-2 epidemic, some of whom lost their lives in doing so. We thank those who have given great and selfless support to the fight against the virus. We thank Ms. Delia Cosgrove and Ms. Ursula Schrammel for the language correction of this manuscript.

\section{Funding}

This work is supported by the National Natural Science Foundation of China (81861138044, 91742114 and 91642118), the National Science and Technology Major Project (2018ZX10723203,
2018ZX10302206, 2017ZX10202201, 2017ZX10202202 and 2017ZX10202203), the Innovation Team Project of the Health Commission of Hubei Province (WJ2019C003), the Fundamental Research Funds for the Central Universities (2020kfyXGYJ016 and 2020kfyXGYJ028), the Integrated Innovative Team for Major Human Diseases Program of Tongji Medical College and the "Double-First Class" Project for the International Cooperation Center on Infection and Immunity, HUST, and a special joint project of University Hospital Essen, University of Duisburg-Essen. The funding sources of this study did not play any role in the study design, the collection, analysis and interpretation of the data, in the writing of the manuscript and in the decision to submit the paper for publication.

\section{Supplementary materials}

Supplementary material associated with this article can be found in the online version at doi:10.1016/j.ebiom.2020.102763.

\section{References}

[1] World Health Organization. Coronavirus disease (COVID-19) outbreak situation. 2020. Available at: https://www.who.int/emergencies/diseases/novel-coronavirus-2019. Accessed March 26, 2020.

[2] National Health Commission of the People's Republic Of China. Update on the epidemic situation of novel coronavirus pneumonia as of 24:00 on March 25. Available at: http://www.nhc.gov.cn/xcs/yqfkdt/202003/f01fc26a8a7b48debe194bd1277fdba3. shtml. Accessed March 26, 2020

[3] Huang C, Wang Y, Li X, Ren L, Zhao J, Hu Y. et al. Clinical features of patients infected with 2019 novel coronavirus in Wuhan, China. Lancet 2020;395 (10223):514-23.

[4] Chan JF, Yuan S, Kok $\mathrm{KH}, \mathrm{To} \mathrm{KK}$, Chu $\mathrm{H}$, Yang J et al A familial cluster of pneumonia associated with the 2019 novel coronavirus indicating person-toperson transmission: a study of a family cluster. Lancet 2020;395 (10223):514-23.

[5] Wang D, Hu B, Hu C, Zhu F, Liu X, Zhang J. et al. Clinical characteristics of 138 hospitalized patients with 2019 novel coronavirus-infected pneumonia in Wuhan, China. JAMA 2020;395(10223):514-23.

[6] de Wit E, van Doremalen N, Falzarano D, Munster VJ. SARS and MERS: recent insights into emerging coronaviruses. Nat Rev Microbiol 2016;14(8):523-34

[7] Chien JY, Hsueh PR, Cheng WC, Yu CJ, Yang PC. Temporal changes in cytokine/chemokine profiles and pulmonary involvement in severe acute respiratory syndrome. Respirology 2006;11(6):715-22.

[8] Chen N, Zhou M, Dong X, Qu J, Gong F, Han Y. et al. Epidemiological and clinical characteristics of 99 cases of 2019 novel coronavirus pneumonia in Wuhan, China: a descriptive study. Lancet 2020:395(10223):507-13.

[9] Qin C, Zhou L, Hu Z, Zhang S, Yang S, Tao Y. et al. Dysregulation of immune response in patients with COVID-19 in Wuhan, China. Clin Infect Dis 2020 published online Mar 12. doi: 10.1093/cid/ciaa248.

[10] Chen G, Wu D, Guo W, Cao Y, Huang D, Wang H. et al. Clinical and immunologic features in severe and moderate coronavirus disease 2019. J Clin Invest 2020 published online Apr 13. doi: 10.1172/JCI137244.

[11] Chang D, Lin M, Wei L, Xie L, Zhu G, Dela Cruz CS. et al. Epidemiologic and clinical characteristics of novel coronavirus infections involving 13 patients outside Wuhan, China. JAMA 2020;323(11):1092.

[12] Chu H, Zhou J, Wong BH, Li C, Cheng Z, Lin X. et al. Productive replication of middle east respiratory syndrome coronavirus in monocyte-derived dendritic cells modulates innate immune response. Virology 2014;454:197-205.

[13] Zhou J, Chu H, Li C, Wong BH, Cheng ZS, Poon VK. et al. Active replication of middle east respiratory syndrome coronavirus and aberrant induction of inflammatory cytokines and chemokines in human macrophages: implications for pathogenesis. J Infect Dis 2014;209(9):1331-42.

[14] Kong SL, Chui P, Lim B, Salto-Tellez M. Elucidating the molecular physiopathology of acute respiratory distress syndrome in severe acute respiratory syndrome patients. Virus Res 2009;145(2):260-9.

[15] Kim KD, Zhao J, Auh S, Yang X, Du P, Tang H. et al. Adaptive immune cells temper initial innate responses. Nat Med 2007:13(10):1248-52.

[16] Palm NW, Medzhitov R. Not so fast: adaptive suppression of innate immunity. Nat Med 2007;13(10):1142-4

[17] Liu J, Liu Y, Xiang P, Pu L, Xiong H, Li C. et al. Neutrophil-to-lymphocyte ratio predicts severe illness patients with 2019 novel coronavirus in the early stage. medRxiv 2020 [Preprint]. February 12, 2020 [cited 2020 Feb 16]. Available from:. doi: $10.1101 / 2020.02 .10 .20021584$

[18] Wang F, Nie J, Wang H, Zhao Q Xiong Y, Deng L. et al. Characteristics of peripheral lymphocyte subset alteration in COVID-19 pneumonia. J Infect Dis 2020 published online Mar 30. doi: 10.1093/infdis/jiaa150.

[19] Channappanavar R, Perlman S. Pathogenic human coronavirus infections: causes and consequences of cytokine storm and immunopathology. Semin Immunopathol 2017;39(5):529-39. 